\title{
On the fictional and real lost gravitation force
}

\author{
Zdzislaw Pluta, Tadeusz Hryniewicz* \\ Faculty of Mechanical Engineering , Koszalin University of Technology, \\ Raclawicka 15-17, PL 75-620 Koszalin, Poland \\ *E-mail addres: Tadeusz.Hryniewicz@tu.koszalin.pl
}

\author{
"Search for the truth of bright flame! \\ Search for new, undiscovered tracks ..." \\ Adam Asnyk
}

\begin{abstract}
The paper covers a critical approach to the natural reality study which is the method of scientific cognition. The existent so called d'Alembert's rule is the link of reality on which the specific attention is focused. The critics is directed to the fictional inertia force covered in the mentioned rule. The necessity for its elimination from the reality description has been presented. Instead, it is proved that a real inertia force occurs. A proper exemplification material has been delivered with two examples of force equilibrium of the system. One of them refers to a rigid system being both under stable and unstable states. The second one covers also these two states but it is referred to a mass-flexible system. Finally, the discussed solution of the problem has been explained on the example of mathematic pendulum and a mass-string system.
\end{abstract}

\section{Keywords:}

Fictional gravitation force; d'Alembert's rule; Real gravitation force/gravitation force; Stable static potential field (SSPF); Unstable static potential field (ASPF); Interstate space; Space-time; Rigid system; Mass-flexible system

\section{INTRODUCTION}

In the universe, which may be considered as a system being under the dynamic equilibrium, each body or a planet can be analysed in a specific sub-system [1]. It may be referred to both macro-, meso- and micro-system [1,2]. Some centuries ago the d'Alembert's rule was announced with the approach consciously filled with a specific imagination. That way the dynamic material system was to be considered as a system in equilibrium. However, because the real dynamic conditions of motion were not in equilibrium, d Alembert decided to introduce a mystification theory which assumed the equilibrium of reality and fiction. Thus until now this theory is in force, grounded and treated like a paradigm.

There is no need to develop the paradigm of the d'Alembert's rule. It is well known, common and ongoing in use. Even the latest reference like e.g. [2] presents it criticlessly, without any shadow of doubt. However, there is another pole of this matter, a critical one $[3,4]$, with this work being destined to that place. 
Not developing too much that fictional aspect of the problem one needs to note that the rule assumes in fact the equilibrium of changes: real forces, and the fictional inertia force. In describing the reality one does not need to say of the real forces as all they are the measures of real mutual interactions of the material bodies. Therefore there are different forces with the names ascribed to them for determined reasons, e.g. the gravitation force, the flexible resistance force, and the thrust force. There is also an inertia force to be included for the considerations.

Finally, it is worth mentioning that there are some signs proving of the necessity to change the approach to study the natural reality meaning the method of scientific cognition. For instance, it appears the non-linear vibrations discussed in [5] is still open. Here the philosophy of approach to the inertia force is presented, indicating a necessity to enter the „black box" in view of solving the adequacy of the model to reality.

Following the earlier publications [6-9], the Authors of the work wish to influence a further changes, advantageous to science. Let this work, treating about fictional and real lost inertia force, proves of the adequate approach.

In the paper, the existence of a real inertia force has been explained on two essential examples with the force equilibrium of the system. One of them is concerned on a rigid system, being under stable and unstable states. The second one covers also these two states but in reference to the mass-flexible system.

\section{STABLE AND UNSTABLE STATES OF A RIGID SYSTEM}

Let a material body of weight $Q$ rests on a determined base (Fig. 1a). It is under a stable state determined by the statics (referred to the third Newton's law) as the force equilibrium, expressed by the equality of two forces running in opposite directions. It is about the gravity force $Q$ (the gravity force of Earth) and the reactive force of base, that is $R$. Therefore, finally $Q=R$.

It is worth noting that the considered system in fact consists of three bodies: the base, the body resting on it, and the Earth. Thus three forces are to be existent here, resulting from the definition of force as the measure of the mutual interaction of material bodies (so many bodies as many forces). Therefore the question arises: where is the third force? That is the inertia force $B_{o}$, which in this stable state co-exists with the gravity force, that may be recorded as follows: $Q \otimes B_{o}$. That way determined the stable equilibrium form a deeper established balance as the energetic equilibrium. It may be recorded as follows:

$$
R=Q \otimes B_{o}
$$

and that in turn corresponds with two equalities of this kind:

$$
R=Q ; R=B_{o}
$$

Thus as visible, the energetic equilibrium discloses the third force, being the inertia force. That last force is quite well visible in the unstable system, where the force equilibrium contains just the three forces, with the reaction force being substituted by an active force $F$ (see Fig. 1). Therefore 


$$
F=Q+B_{o}
$$

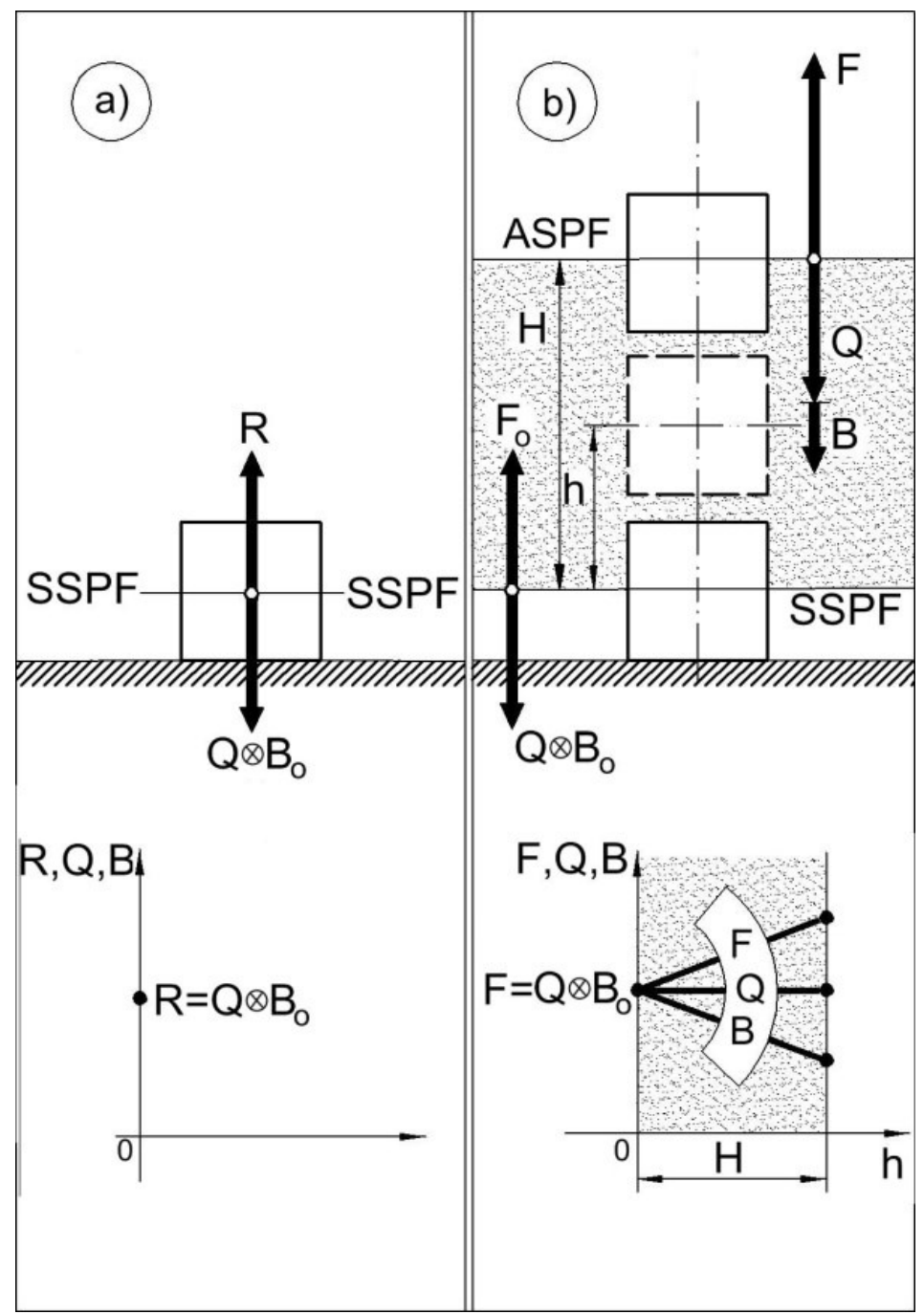

Fig. 1. Stable and unstable states of a rigid system

The courses of all these forces on the way $H$ of the body climb/ascent have also been presented in Fig. 1b. The gravity force $Q$ does not undergo any change, and is constant; the force $F$ rises properly, and the gravity force undergoes diminution.

From the energetic point of view the considered body was at first on a stable static potential field (SSPF), and after transition through the interstate space (dotted zone in Fig. 1) it occupied the neighbouring, unstable static potential field (ASPF). Energetic states of the body are equal on the both fields. That means the sum of potentials, as the measure of particular energies, does not undergo any change.

It is worth noticing that the transition of a body from the stable state to an unstable state reveals the inertia force (that real one) and disturbs the force equilibrium. Thus in the interstate space the body dynamics, fully and with proper meaning of the word, takes place. Not earlier than on the neighbouring potential field again the force equilibrium takes place but it covers the revealed inertia force; it has been different both quantitatively and qualitatively. One should note that the notion of the interstate space introduced herewith does not take into 
account the time factor, resulting in rectilinear courses of the forces. The gravity force is an exception as it does not undergo any change in time. The framework of this paper does not cover the time courses (due to the description of the magnitudes as the function of time) which take place in the space-time, the time-space outgrowth. Such a formal differentiation of these notions seems to be justifiable.

\section{MASS-FLEXIBLE SYSTEM AND ITS STATES: STABLE AND UNSTABLE}

The mass-flexible system (Fig. 2) allows for a decisive proof for existence of the inertia force. Lifting a body by means of a pull spring allows to confirm the variability of active force $F$, equal in this case the sum of the forces $S$ and $R$.

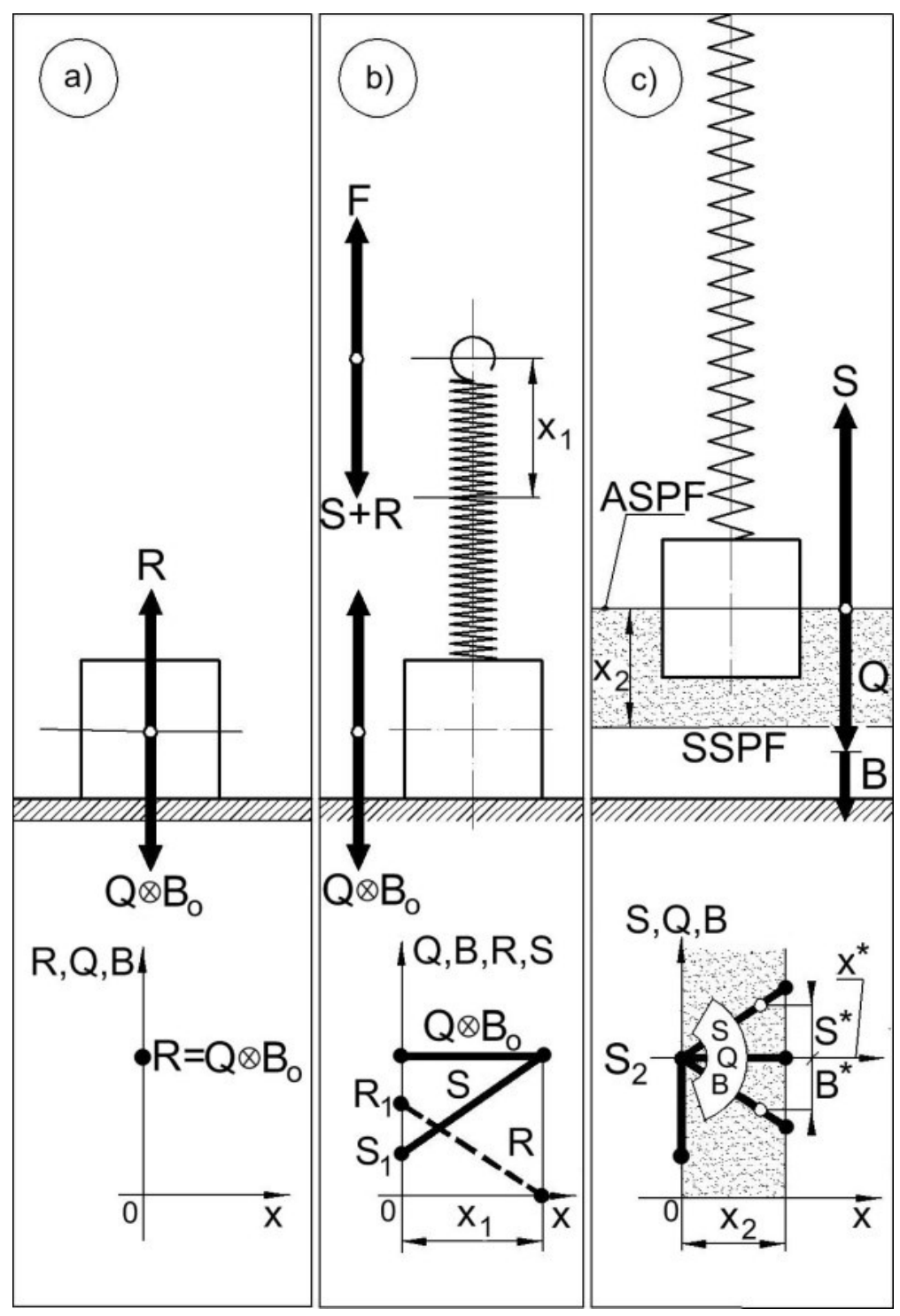

Fig. 2. Mass-flexible system and its energetic states

In the first position of a body (Fig. 2a) there is no external stimulus acting on it. The body is placed here on a stable static potential field (SSPF), that corresponds with the force equilibrium $R=Q$, and the energetic one of the form: $R=Q \otimes B_{o}$. 
Further on, the external stimulus begins to act there (Fig. 2b) and the sign of that reaction is the pull spring tension. However, the spring does not undergo an elongation at the beginning due to its initial tension. Therefore at the beginning of external loading of the body when $S=S_{l}$, the reaction force of the base $R_{l}$ equals

$$
R_{1}=Q-S_{1}
$$

From that moment (on the way $x_{1}$ ) these forces undergo changes: the force $S$ rises, and the force $R$ decreases, respectively. All the time the energetic state of the body remains invariable in spite of the changes of the higher mentioned forces. Here their sum $(S+R)$ is constant and balances the gravity force $Q$, which in turn still co-exists with the inertia force $B_{o}$, i.e. $Q \otimes B_{o}$. That all may be written as follows:

$$
S+R=Q \otimes B_{o}
$$

A neuralgic state of the body takes place in case if $R=0$, and $S=Q \otimes B_{o}$. From this moment the body is getting to the interstate space of the length $x_{2}$ (dotted place in Fig. 2). There the gravity force still remains constant/invariable, the flexible force $S$ rises on and on, with the value rising from $S_{1}$ to $S_{2}$ at $x_{2}=0$ and the inertia force $B$ is now decreasing (Fig. 2c). Here is no force equilibrium. It appears not earlier than on the neighbouring unstable static potential field (ASPF). That equilibrium takes the following form:

$$
Q+B=S
$$

That is the adequate description of the considered part of reality. Here is no fictional force, instead here is a real force of that kind, visible, because considered in a proper coordinate system, connected with the beginning of the phenomenon of the body transition from one to another energetic state.

The transfer of the co-ordinate system to the place of beginning of the changes of flexible force, the introduction of fictional inertia force, the differential record of the equity of changes of both forces - all they are not in agreement with the main assignment of science which should be directed to the description of reality. In this light, the creation of fictionalreal descriptions has no scientific reason.

In Fig. 2 the position of the transferred point $0^{*}$ of the co-ordinate system has been also marked. In accordance to the d'Alembert's rule in such a co-ordinate system the so called dynamic equilibrium occurs. It appears here that the flexible force $S^{*}$ is in fact just the increment of that kind of force, and the fictional inertia force $B^{*}$ is something that does not exist, being - as the classical mechanics says - a kind of the auxiliary structure.

\section{ENERGETIC STATES OF PENDULUM}

Let us the following illustration example be a mathematic pendulum (Fig. 3). A body hung on a string of length $l$ appears first on a stable static potential field (SSPF), being with this at a stable state (Fig. 3a). In this state the inertia force $B_{o}$ is the biggest and co-exists with the gravity force $Q$, so $Q \otimes B_{o}$. Now the string reacts on the body with the tension force $S_{o}$, equal with the same to the forces $Q$ and $B_{o}$. Therefore $S_{o}=Q \otimes B_{o}$. 
At the moment of appearance of an external stimulus which with the force $F_{o}$ (Fig. 3b) attempts to bring the body out of the stable equilibrium, a body resistance appears, resulting from its inertia. A measure of that resistance is the inertia force, here the initial inertia force $B_{s}^{o}$. At the first moment it equalizes the mentioned active force $F_{o}$. Thus $F_{o}=B_{s}^{o}$. In that situation the second stable static potential field begins to act.

Now the body is getting through two spaces (dotted fields in Fig. 3), along with the trajectory being the segment/arc of the circle in the range of angle $\alpha_{o}$ (Fig. 3c). One of them is a gravity-inertia space, the second one (that one on the angular direction) has an inertia nature. By the external action with the force $F_{\alpha}$ the body reaches the unstable static potential fields (ASPF), which close the both mentioned spaces.

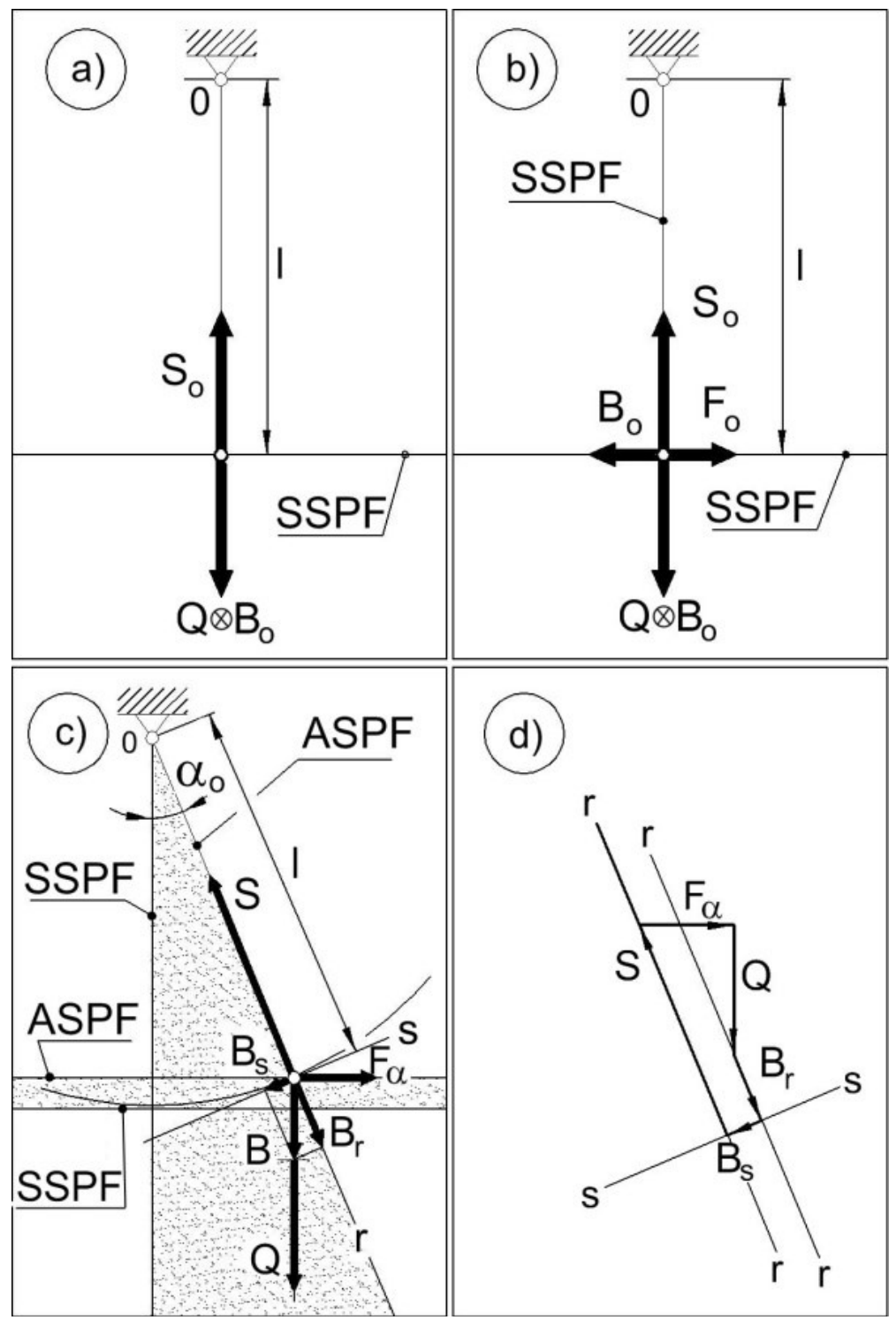

Fig. 3. Energetic states of mathematic pendulum

It is visible, the gravity force does not change its value, it remains constant all the time and is situated vertically. It does not undergo decomposition into other directions, with no component directions. That is the inertia force (existent, and real) which has determined the components on two mutually orthogonal directions: tangent $s-s$ and radial $r-r$. On the tangent direction there is a component $B_{s}$, and the radial one occupied by the force $B_{r}$. 
All the forces, with the system bodies (a body hung on string, the string, Earth, external stimulus) reacting one on each other, are in equilibrium. The polygon of those convergent forces (Fig. 3d) reflects graphical formulation/presentation of the mentioned equilibrium.

\section{ENERGETIC STATES OF A MASS-STRING SYSTEM}

The mass-string system, not fulfilling the rule of mathematic pendulum, has been the last illustrative example here (Fig. 4). Hung on the string a material body is at first on a stable static potential field (SSPF), and then, by means of a determined external stimulus, is transferred on the inclined plane (Fig. 4a). There it is under an unstable state, being on the unstable static potential field (ASPF).

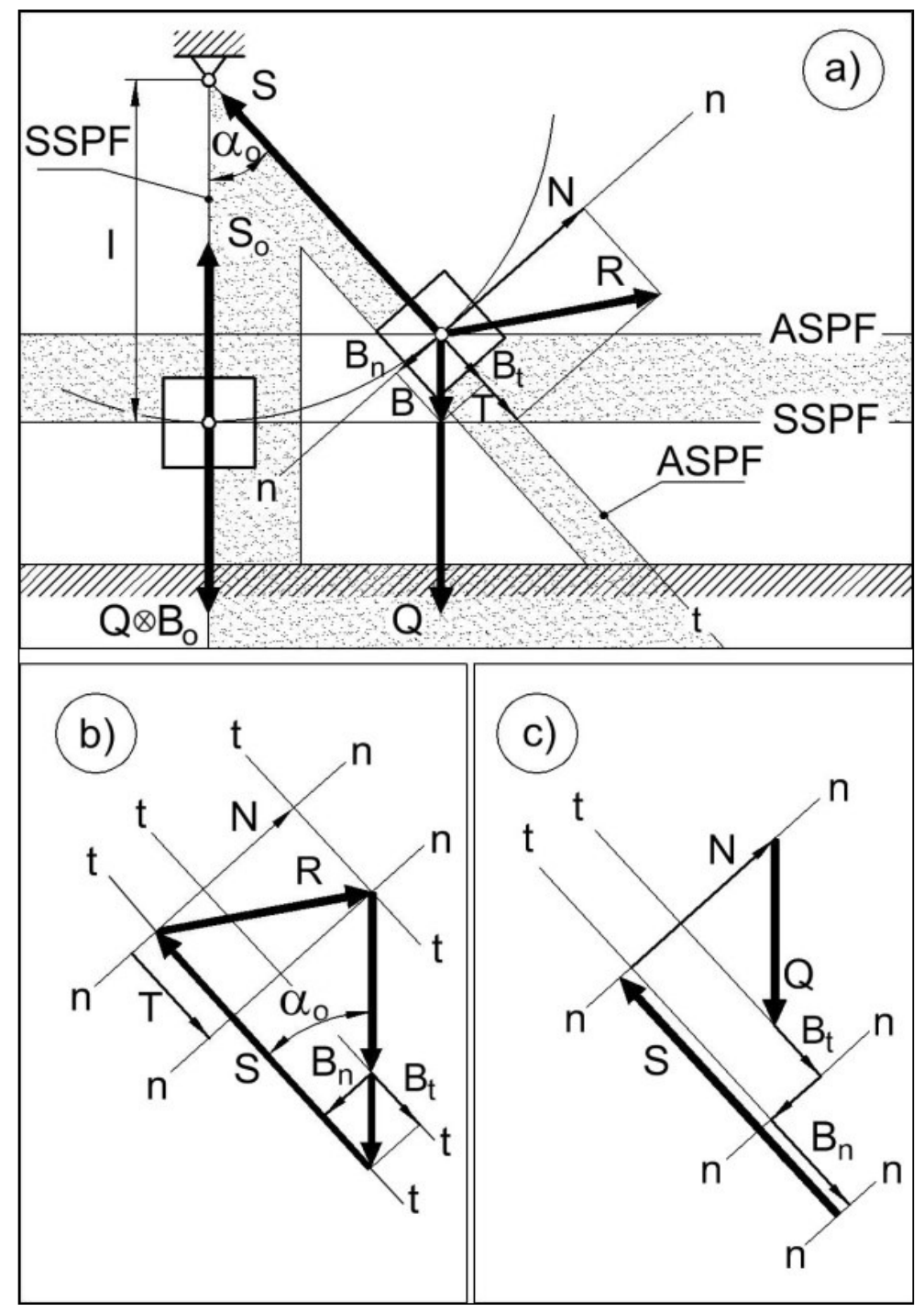

Fig. 4. Energetic states of mass-string system

As can be noticed, the stable states of this and former systems (see Fig. 3) do not differ at all. However, the unstable states are described differently. In the last case (Fig. 4a) the role of force $F_{\alpha}$ has been taken over by the reaction force of the inclined plane, i.e. $R$. There are the following components of the force: tangent $T$, being the force of surface resistance; and normal $N$, which is perpendicular to the oblique surface of the wedge. The remaining forces 
do not require any explanations, as they are analogous as in reference to the mathematic pendulum.

All the forces, with the system bodies (a body hung on string, the string, Earth, the inclined plane) reacting one on each other, are in equilibrium. The polygon of those forces (Fig. 4b) reflects the graphical formulation/presentation of the equilibrium with the thick vectors referred to the essential forces and the thin vectors illustrating the force components of: reaction $R$, and inertia $B$. The polygon of forces (Fig. 4c) is properly developed and presents a closed system of two essential forces $(Q, S)$ and those which are under these circumstances the components of others.

\section{SUMMARY}

It results from the presented considerations that the inertia force, being a measure of the body inertia, is and exists in reality. At a stable static state it co-exists with the gravity force in vertical direction and in horizontal direction it appears at the moment of the beginning of action a determined external stimulus on the body. The inertia force has always such a sense which indicates tending the body to the stable state in accordance with the nature of the body as the general natural law which expresses the nature.

The classical mechanics does not take into account these two static states as it does not notice the body inertia in these conditions. It appears, as indicated above, there are two static states: stable and unstable. Of course, the transition from one state to another is possible only through the interstate space, existing between the potential fields on which the body takes over a determined energetic state.

The subject presented in the work refers only to the quest of existence of the body inertia on the static potential fields, as well as between these fields, that is in the interstate space. It is worth noting that in the uniform (also rectilinear) motion there is also an inertia, because under these conditions the acceleration is positive, greater than zero. However, that is not the subject of this work and will not be considered and developed herewith.

The last of the mentioned problems is to be developed by the Authors in a near future. Also many other problems will be discussed in view of developing just mentioned here some real quests of the mechanical reality. A new in-depth approach is required to describe the body dynamics which is the motion in the space-time. The presented here the energetic states of a material body, being also in the mathematic pendulum system [6-9], open a perspective of creation the adequate theory of such a pendulum. All these efforts are made to prove that all the natural reality has a quantum nature.

From these considerations, devoted to the subject of a material body inertia, a constitutive conclusion appears that the inertia is an immanent feature of the body. Thus it appears there is no justification for introducing a fictional inertia force to the system and creating a real-fictional hybrid. Such a direction of activities is a sort of sophisticated speculation. The adequate description of the reality, taking into consideration the immanent feature of a material body which is the inertia, should be included to the structure of science. This feature with the measure being the inertia force, just real not a fictional one, is of importance in our approach . 


\section{REFERENCES}

[1] Tomasz Borowski, International Journal of Chemistry, Physics and Astronomy 1 (2012) $1-5$.

[2] J.R. Taylor, Classical mechanics (Mechanika klasyczna), Vol. 1 and 2, (transl from English). 1st editio, Wydawnictwo Naukowe PWN SA, Warszawa 2006.

[3] Zdzisław Pluta, LAB 2 (2004) 50-52.

[4] Zdzisław Pluta, LAB 6 (2004) 42-46.

[5] Z. Dąbrowski, Przegląd Mechaniczny 11 (2007) 15-18.

[6] Zdzisław Pluta, Tadeusz Hryniewicz, International Journal of Chemistry, Physics and Astronomy 2 (2012) 28-34.

[7] Zdzisław Pluta, Tadeusz Hryniewicz, International Journal of Chemistry, Physics and Astronomy 3 (2012) 1-10.

[8] Zdzisław Pluta, Tadeusz Hryniewicz, International Journal of Chemistry, Physics and Astronomy 3 (2012) 11-23.

[9] Zdzisław Pluta, Tadeusz Hryniewicz, International Journal of Chemistry, Physics and Astronomy 4 (2012) 1-7. 